\title{
Use of washing cytology from removed self-expandable metal stents for biliary strictures: a novel cytology method $\square$
}

\section{다)(1) $\odot(9)$}

\author{
Authors

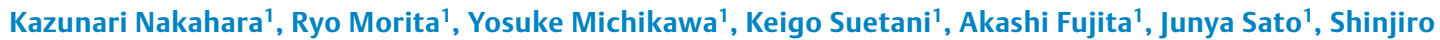 \\ Kobayashi $^{2}$, Akira Endo ${ }^{3}$, Yasushi Ariizumi ${ }^{3}$, Junki Koike ${ }^{3}$, Masayuki Takagi ${ }^{3}$, Takehito Otsubo ${ }^{2}$, Fumio Itoh ${ }^{1}$
}

Institutions

1 Department of Gastroenterology and Hepatology, St. Marianna University, School of Medicine, Kawasaki, Japan

2 Department of Gastroenterogical and General Surgery, St. Marianna University School of Medicine, Kawasaki, Japan

3 Department of Pathology, St. Marianna University, School of Medicine, Kawasaki, Japan

submitted 5.11.2019

accepted after revision 17.2.2020

\author{
Bibliography \\ DOI https://doi.org/10.1055/a-1144-2668 | \\ Endoscopy International Open 2020; 08: E748-E752 \\ (c) Georg Thieme Verlag KG Stuttgart · New York \\ eISSN 2196-9736
}

Corresponding author

Kazunari Nakahara, Department of Gastroenterology and Hepatology, St. Marianna University School of Medicine, 2-16-1, Sugao, Miyamae-ku, Kawasaki, 216-8511, Japan Fax: +81-44-976-5805

nakahara@marianna-u.ac.jp

\section{ABSTRACT}

Removability is one of the important features of biliary covered self-expandable metal stents (CSEMS). In this study, we evaluated the diagnostic ability of washing cytology of removed CSEMS. For 14 removed CSEMS that had been placed for the biliary strictures (12 malignant, 2 benign), the surface of CSEMS was washed with saline, and pathological examination of the washing liquid as cytology (CSEMS washing cytology) was performed. The specimen sampling rates and sensitivity for malignancy of CSEMS washing cytology were $92.9 \%$ and $41.7 \%$, respectively. Sensitivity according to the primary disease was $60.0 \%$ for bile duct cancer and $20 \%$ for pancreatic cancer. Sensitivities based on the methods of stent removal were $16.7 \%$ and $66.7 \%$ for removal through the channel of the scope and with the scope, respectively. Therefore, it is possible that sensitivity of CSEMS washing cytology is higher in bile duct cancer and for removal with the scope. In conclusion, CSEMS washing cytology may have potential as a pathological diagnostic method.

\section{Introduction}

Generally, biliary stricture is pathologically diagnosed by bile cytology and biliary forceps biopsy with endoscopic retrograde cholangiopancreatography (ERCP). However, the sensitivity (cancer detection rate) for malignant biliary stricture is reported to be $30 \%$ to $57 \%$ with brush cytology and $43 \%$ to $81 \%$ with biliary biopsy [1], indicating that in many instances, pathological diagnosis is difficult. When the pathological diagnosis of malignancy cannot be obtained, diagnosis must need to be determined based on imaging findings and clinical course, and then, a metal stent may be placed.

In recent years, covered self-expandable metal stents (CSEMS) have been reported to be safely removed [2,3], mak- ing CSEMS even more useful from the perspective of reintervention. Removed CSEMS are generally simply discarded. However, we washed the surface of a removed CSEMS with saline considering the possibility that tumor cells or tissues might be attached to the stent surface in contact with the lesion, and pathological examination of the washing liquid as cytology (CSEMS washing cytology) was performed. In the current study, we evaluated the diagnostic performance of CSEMS washing cytology for biliary strictures. 


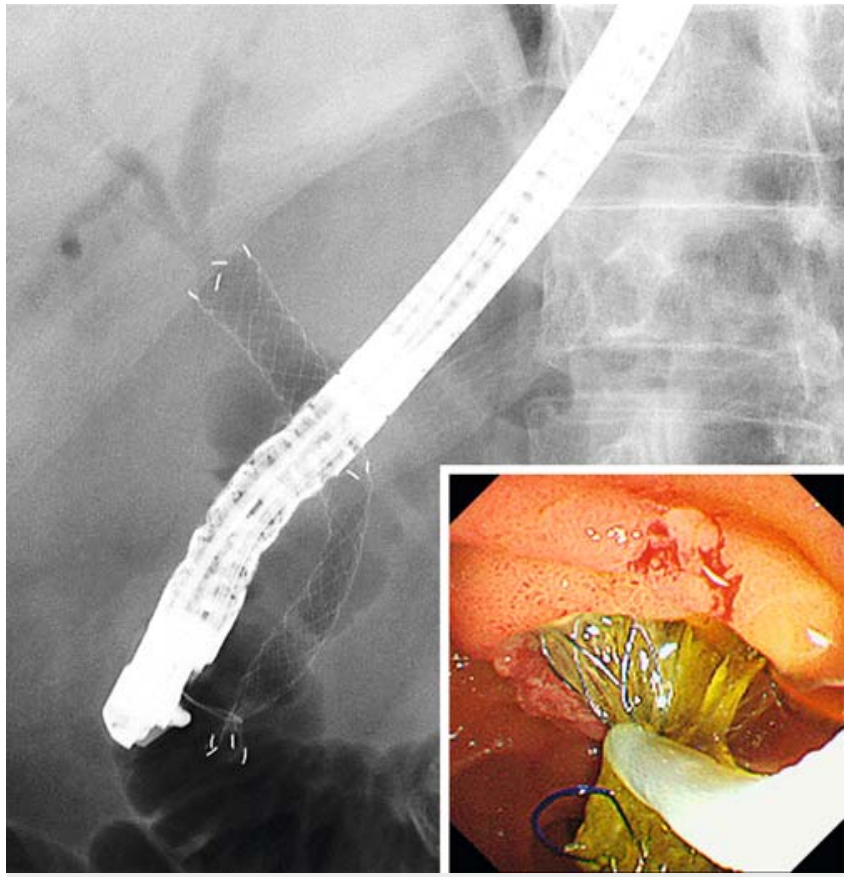

- Fig. 1 Removal of a covered self-expandable metal stent using a polypectomy snare.

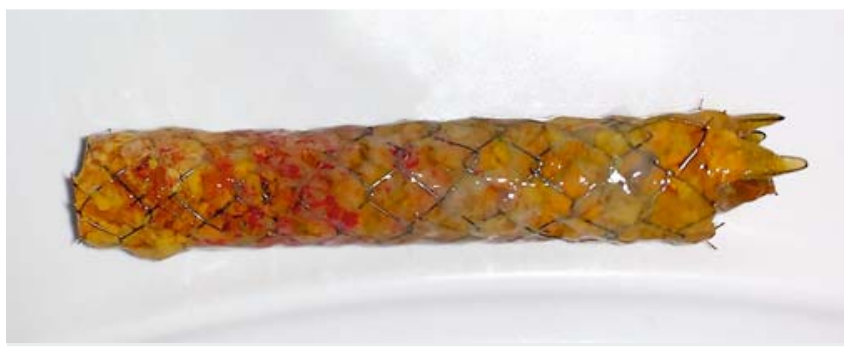

- Fig. 2 A removed covered self-expandable metal stent.

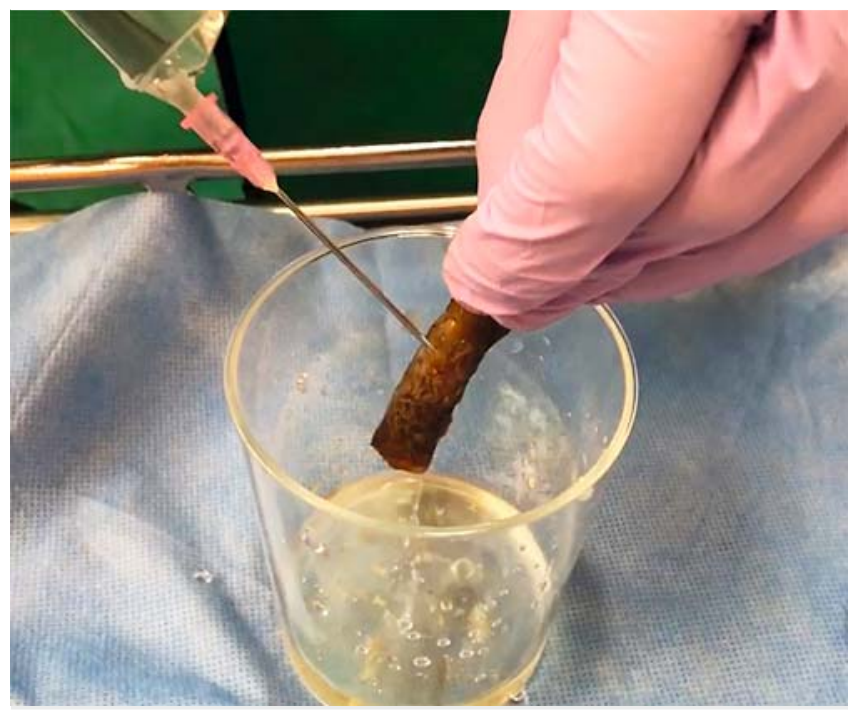

- Fig. 3 Technique for washing cytology with covered self-expandable metal stents. While spraying with saline, tissue attached to the surface of the removed stent was washed and scraped off with a 19-gauge needle.

\section{Patients and methods}

\section{Patients}

The subject sample included 14 sessions of CSEMS washing cytology in 11 patients [males: 9, females: 5, age: $69.0 \pm 15.0$ years (mean \pm standard deviation)] whereby CSEMS for distal biliary stricture was removed and CSEMS washing cytology was performed at our hospital between June 2017 and September 2018. In three of 11 patients, CSEMS washing cytology was performed twice. The primary diseases of distal biliary strictures were bile duct cancer in five patients, pancreatic cancer in five, lymph node metastasis in two, and chronic pancreatitis in two.

Endoscopic sphincterotomy (EST) was performed before CSEMS placement in 12 patients. All CSEMS were placed across the papilla. CSEMS were of the fully covered type in 11 patients [Hanaro stent (M.I. Tech, Seoul, South Korea): five, Niti-S ComVi stent (Taewoong Medical Inc., Goyang, South Korea): three, and WallFlex Biliary RX stent (Boston Scientific, Marlborough, Massachusetts, United States): three] and of the partially covered type in three patients (WallFlex Biliary RX stent: three). The diameter of CSEMS was $10 \mathrm{~mm}$ for all patients, with a length of $6 \mathrm{~cm}$ in seven patients, $7 \mathrm{~cm}$ in four patients, and 8 $\mathrm{cm}$ in three patients.

The reasons for CSEMS removal were stent obstruction in eight patients, concurrent acute cholecystitis in two patients, concurrent acute pancreatitis in one patient, and re-evaluation of stricture in three patients. The median duration of CSEMS placement was 73.5 (range, 2-116) days.

In the same ERCP session where CSEMS washing cytology was performed, bile aspiration cytology was also performed in three patients. Prior pathological examinations by ERCP included bile cytology in 12 patients (brush cytology in nine and aspiration cytology in three) and biliary biopsy in 10 patients (some patients overlapping).

\section{CSEMS removal}

We used a duodenoscope [T]F260V (Olympus Medical Systems Corp., Tokyo, Japan)]. The CSEMS was grasped using a snare ( $\triangleright$ Fig. 1); if possible, the CSEMS was removed through the channel of the scope and if not, it was removed together with the scope while being grasped with the snare. The CSEMS was removed through the channel in eight and with the scope in six. A removed CSEMS is shown in - Fig. 2.

\section{Techniques for CSEMS washing cytology}

A 19-guage needle was mounted on a syringe containing saline. While spraying with saline, tissue attached to the surface of the CSEMS was washed and scraped off ( $\triangleright$ Fig. $\mathbf{3}$, $\triangleright$ Video $\mathbf{1}$ ). The stent washing liquid was submitted for pathological examination as a specimen for cytology. The sample of stent washing liquid is shown in > Fig. 4.

\section{Pathological diagnosis}

Specimens were considered to be malignant when malignant cells were clearly identified (Class V) or when highly atypical cells suspected to be malignant were observed (Class IV). Samples of cytologic specimen indicating adenocarcinoma are 
shown in - Fig. 5. Specimens were considered to be benign if normal cells were identified, or atypical cells which cannot rule out malignancy were found.

\section{Measurements}

We retrospectively examined the specimen sampling rates, sensitivity, and specificity of CSEMS washing cytology. We also evaluated the relationship between CSEMS washing cytology results and patient backgrounds or stent removal techniques. Furthermore, we compared results of CSEMS washing cytology with those of other pathological examinations.

The specimen sampling rate was defined as the rate of obtaining specimens in adequate quantity and quality for diagnosis. Sensitivity indicates the cancer detection rate.

This study was approved by the institutional review board of St. Marianna University School of Medicine.

\section{Statistical analysis}

Fisher's exact test and Mann-Whitney $U$ test were used for statistical analysis, where appropriate. $P<0.05$ was considered to be significant. Statistical analysis was performed using StatMate IV software (ATMS Co., Ltd., Tokyo, Japan).

\section{Results}

In CSEMS washing cytology, the specimen sampling rates was $92.9 \%(13 / 14)$ and the sensitivity was $41.7 \%$ (5/12). Of the two cases with benign stricture of chronic pancreatitis, one had no specimen. Sensitivities based on the primary disease were $60.0 \%$ (3/5) for bile duct cancer, $20 \%$ (1/5) for pancreatic cancer, and $50.0 \%$ (1/2) for lymph node metastasis. Although there was no statistical difference $(P=0.519)$, sensitivity was the highest for bile duct cancer. In the malignant cases, median stent placement duration was 71 (range, 2-116) days for five patients in whom malignancy could be diagnosed by CSEMS washing cytology and 76 (range, 15-113) days for seven patients in whom malignancy could not be diagnosed, with no significant difference. Sensitivities based on the method of stent removal was $16.7 \%(1 / 6)$ and $66.7 \%(4 / 6)$ for stents removed through the channel of the scope and for those removed with the scope, respectively. Although no statistical difference was observed $(P=0.242)$, sensitivity was higher for stents removed with the scope.

In three patients with malignancy in whom CSEMS washing cytology and conventional aspiration bile cytology were performed in the same ERCP session, malignancy was not diagnosed by conventional bile cytology in all patients (Class III: 2; Class I: 1); however, malignancy could be diagnosed by CSEMS washing cytology in two of the three patients (Class V: 1 , Class IV: 1 , and Class I: 1).

Sensitivities of pathological examination performed in the previous ERCP was $50.0 \%$ (5/10) and $25.0 \%$ (2/8) for conventional bile cytology and biliary biopsy, respectively. Among the five patients in whom malignancy could not be diagnosed by prior pathological examinations, malignancy could be diagnosed by CSEMS washing cytology in two patients. In the malig-

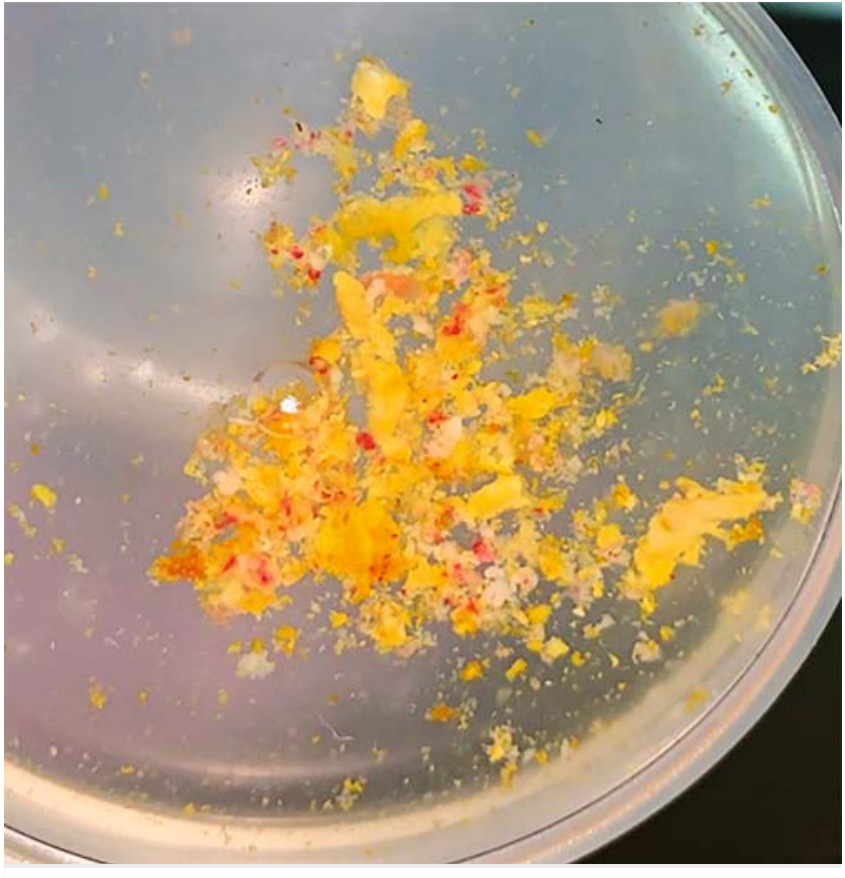

- Fig. 4 Macroscopic findings from the stent washing liquid. Reddish specimens along with debris were observed.

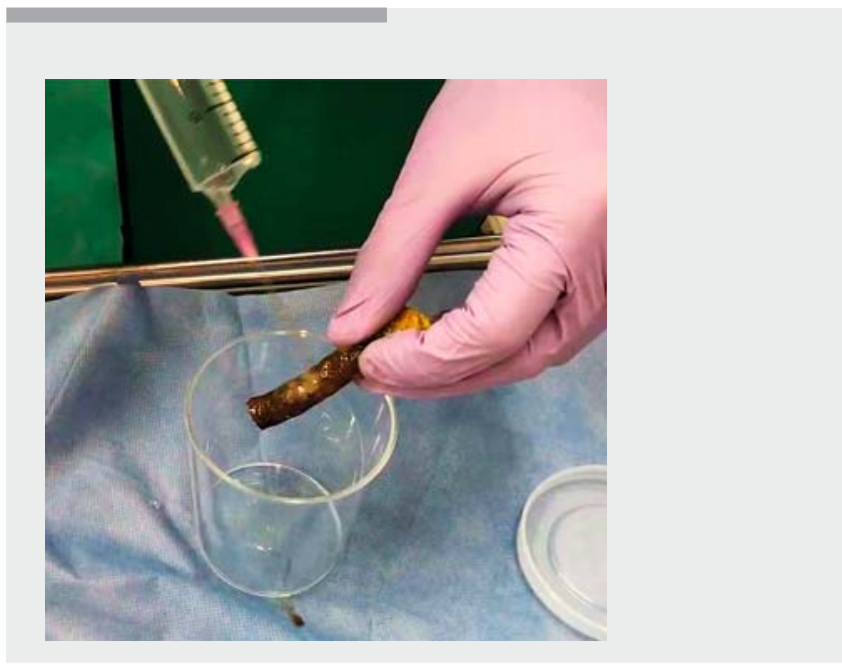

Video 1 Technique of covered self-expandable metal stent washing cytology.

nant cases, the median observation period following CSEMS washing cytology was 168 (range: 8-331) days.

The patient summary is shown in $>$ Table 1.

\section{Discussion}

Herein we report CSEMS washing cytology as a novel pathological diagnostic method for biliary stricture. We found that cellular components were attached to the surface of the removed CSEMS and that specimens could be collected in a state that could be pathologically evaluated. Sensitivity of CSEMS washing cytology was $41.7 \%$, which was neither inferior to the sensi- 


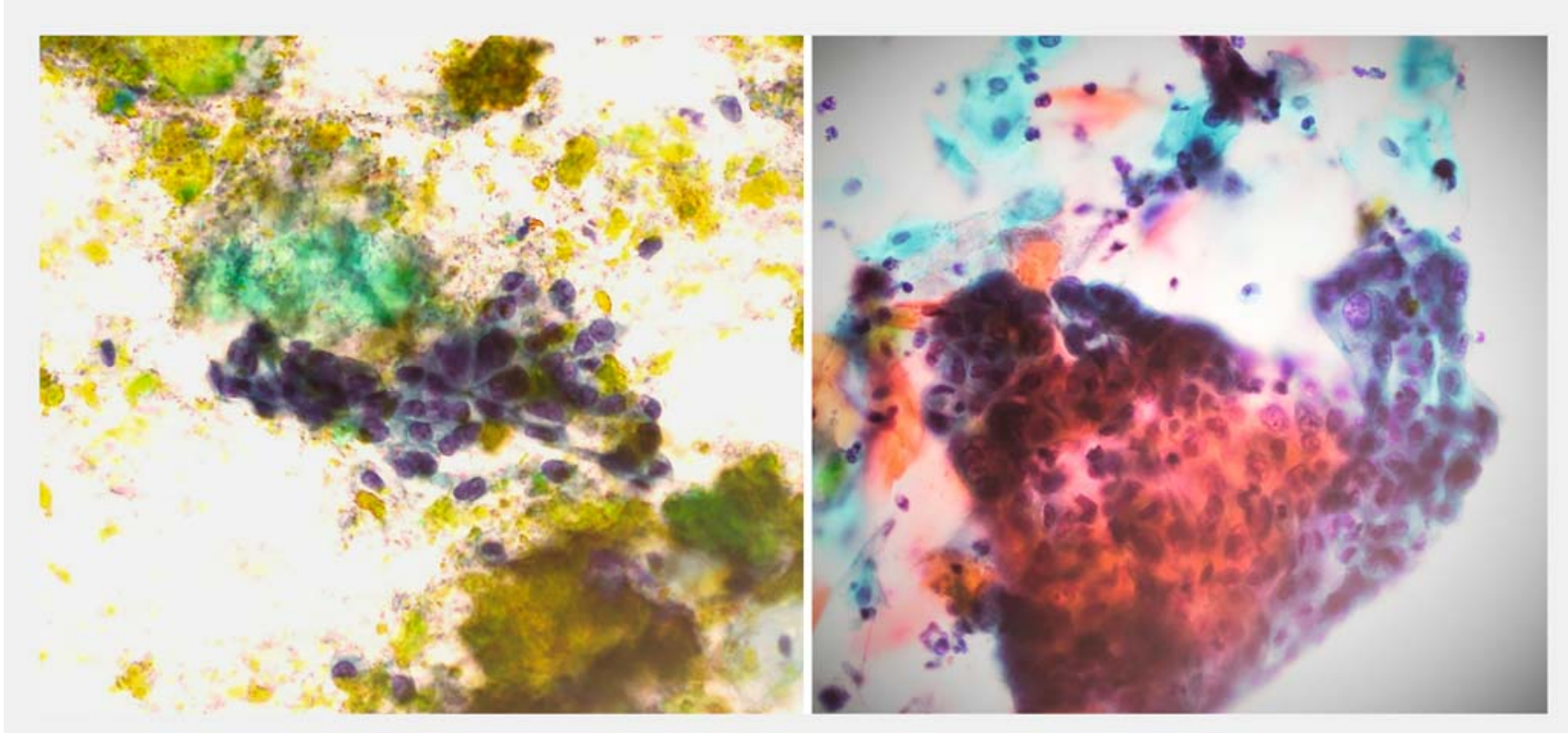

- Fig. 5 Cytologic specimen from the stent washing liquid. Cytological findings indicated adenocarcinoma.

\begin{tabular}{|c|c|c|c|c|c|c|c|c|}
\hline Case & $\begin{array}{l}\text { Primary } \\
\text { disease }\end{array}$ & CSEMS & $\begin{array}{l}\text { Placement } \\
\text { periods (day) }\end{array}$ & $\begin{array}{l}\text { Stent remov- } \\
\text { al method }\end{array}$ & $\begin{array}{l}\text { CSEMS wash- } \\
\text { ing cytology }\end{array}$ & $\begin{array}{l}\text { Simultaneous } \\
\text { bile cytology }\end{array}$ & $\begin{array}{l}\text { Previous bile } \\
\text { cytology }\end{array}$ & $\begin{array}{l}\text { Previous } \\
\text { biliary biopsy }\end{array}$ \\
\hline 1 & $\mathrm{BCa}$ & FC, Niti-S & 78 & WS & Class V & Class III & Class III & No malignancy \\
\hline 2 & LM & FC, Hanaro & 2 & TS & Class IV & - & Class V & Atypical \\
\hline 3 & $\mathrm{BCa}$ & FC, Hanaro & 32 & TS & Class I & Class III & Class II & No malignancy \\
\hline 4 & $\mathrm{BCa}$ & FC, Hanaro & 50 & WS & Class IV & Class II & Class III & Atypical \\
\hline $5-1$ & $\mathrm{PCa}$ & FC, Hanaro & 15 & TS & Class III & - & Class V & Malignancy \\
\hline $5-2$ & PCa & FC, WallFlex & 116 & WS & Class V & - & Class V & Malignancy \\
\hline 6 & LM & FC, Hanaro & 30 & TS & Class V & - & - & - \\
\hline $7-1$ & $\mathrm{BCa}$ & PC, WallFlex & 111 & TS & Class II & - & Class IV & - \\
\hline $7-2$ & $\mathrm{BCa}$ & FC, Niti-S & 71 & WS & Class V & - & Class IV & - \\
\hline $8-1$ & PCa & PC, WallFlex & 76 & WS & Class II & - & Class III & Atypical \\
\hline $8-2$ & PCa & FC, Niti-S & 113 & WS & Class II & - & Class III & Atypical \\
\hline 9 & $\mathrm{PCa}$ & PC, WallFlex & 80 & TS & Class I & - & - & - \\
\hline 10 & $\mathrm{CP}$ & FC, WallFlex & 99 & TS & Class I & - & Class II & Atypical \\
\hline 11 & $\mathrm{CP}$ & FC, WallFlex & 57 & TS & No specimen & - & Class II & No malignancy \\
\hline
\end{tabular}

CSEMS, covered self-expandable metal stent; BCa, bile duct cancer; LM, lymph node metastasis; PCa, pancreatic cancer; CP, chronic pancreatitis; FC, fully covered; PC, partially covered; WS, with the scope; TS, through the scope

tivity previously reported for brush cytology (30\%-57\%) [1] nor inferior to the sensitivity of conventional pathological examinations performed previously for subjects of the current study. Furthermore, malignancy could be diagnosed by CSEMS washing cytology in two of the five patients in whom malignancy could not be diagnosed by prior conventional examinations. Moreover, malignancy could not be diagnosed by bile cytology in any of the three patients in whom CSEMS washing cytology and bile cytology were performed in the same ERCP session; however, diagnosis was achieved in two patients by CSEMS washing cytology. Accordingly, we believe that CSEMS washing cytology has potential as a useful pathological diagnostic method. 
Sensitivities of CSEMS washing cytology based on the primary disease were $60 \%$ and $20 \%$ for bile duct cancer and pancreatic cancer, respectively. Although our evaluation included a small sample size, it is possible that sensitivity is higher in bile duct cancer than in pancreatic cancer. Although endoscopic ultrasound-guided fine-needle aspiration (EUS-FNA) for pancreatic cancer has been reported to have high sensitivity of about $90 \%[4,5]$, EUS-FNA can be technically difficult for bile duct cancer; therefore, CSEMS washing cytology might contribute to better diagnostic modality for bile duct cancer.

No relationship was observed between sensitivity of CSEMS washing cytology and duration of stent placement. We were able to diagnose malignancy even in patients whose stents were removed 2 days after placement. Therefore, we believe that CSEMS washing cytology is worthwhile even for patients whose stents are removed soon after placement.

Sensitivities of CSEMS washing cytology based on the method of stent removal were $16.7 \%$ and $66.7 \%$ for removal through the channel of the scope and that together with the scope, respectively. Although no significant difference was observed because of the small sample size, sensitivity was higher for removal with the scope. When the stent was removed through the channel, cellular components attached to the surface of the stent may have fallen off in the channel, which might have resulted in a lower sensitivity. Therefore, when performing CSEMS washing cytology, removal of the stent together with the scope might be better.

To our knowledge, there are three previous reports on cytology using removed biliary plastic stents (plastic stent cytology) [6-8], but there is no report using removed biliary metal stents except for one case report [9]. According to the initial report on plastic stent cytology, sensitivity for malignancy was high at $79 \%$ [6]. However, in the following two reports with a relatively large number of subjects, sensitivities were low at $36 \%$ [7] and $11 \%$ [8]. As compared with plastic stent cytology, metal stent cytology may allow large amounts of tissue to be sampled, owing to the wide contact area of the stent and the tumor and the rough structure of the stent surface.

The advantage of CSEMS washing cytology is that it is minimally invasive for the patient because the removed stent is washed outside the body to collect a specimen. Therefore, special devices, such as a brush, biopsy forceps, and aspiration needle, are not required to collect the sample, and sample collection can be performed at a very low cost using only an injection needle and saline. Conversely, the limitation is that it does not contribute to early diagnosis because the examination is performed at the time of stent removal. However, in some patients, pathological diagnosis is difficult with conventional methods, and a metal stent is placed without a pathological diagnosis. In recent years, advance in chemotherapy have pro- longed survival and increased circumstances that dictate stent removal/replacement, and to a certain extent, survival can be expected even after reintervention. In the current study, we conducted a median follow-up observation for 168 days after stent removal. Even if it is late, if pathological diagnosis can be achieved with CSEMS washing cytology, we believe that it can be of help to selection of the subsequent treatment plan.

The current study has the following limitations. First, it was retrospective, small and performed at a single institution because it was a preliminary study. Second, because this study contained only a certain kind of CSEMS, it is unclear whether CSEMS washing cytology can be applied to other stents. In future, further examination of many types of stents with a large sample size is desirable.

\section{Competing interests}

The authors declare that they have no conflict of interest.

\section{References}

[1] De Bellis M, Sherman S, Fogel EL et al. Tissue sampling at ERCP in suspected malignant biliary strictures (Part 2). Gastrointest Endosc 2002; 56: 720-730

[2] Kahaleh M, Tokar ], Le T et al. Removal of self-expandable metallic Wallstents. Gastrointest Endosc 2004; 60: 640-644

[3] Kida M, Miyazawa S, Iwai T et al. Endoscopic management of malignant biliary obstruction by means of covered metallic stents: primary stent placement vs. re-intervention. Endoscopy 2011; 43: 10391044

[4] Puli SR, Bechtold ML, Buxbaum JL et al. How good is endoscopic ultrasound-guided fine-needle aspiration in diagnosing the correct etiology for a solid pancreatic mass? A meta-analysis and systematic review Pancreas 2013; 42: 20-26

[5] Banafea O, Mghanga FP, Zhao J et al. Endoscopic ultrasonography with fine-needle aspiration for histological diagnosis of solid pancreatic masses: a meta-analysis of diagnostic accuracy studies. BMC Gastroenterol 2016; 16: 108

[6] Leung JW, Sung JY, Chung SC et al. Endoscopic scraping biopsy of malignant biliary strictures. Gastrointest Endosc 1989; 35: 65-66

[7] Foutch PG, Kerr DM, Harlan JR et al. A prospective, controlled analysis of endoscopic cytotechniques for diagnosis of malignant biliary strictures. Am J Gastroenterol 1991; 86: 577-580

[8] Devereaux BM, Fogel EL, Bucksot L et al. Clinical utility of stent cytology for the diagnosis of pancreaticobiliary neoplasms. Am J Gastroenterol 2003; 98: 1028-1031

[9] Nakahara K, Morita R, Michikawa Y et al. Washing cytology of removed self-expandable metal stent for biliary stricture: A novel cytology technique. Diagn Cytopathol 2019; 47: 743-745 